\title{
Ingesting a pre-workout supplement containing caffeine, B-vitamins, amino acids, creatine, and beta-alanine before exercise delays fatigue while improving reaction time and muscular endurance
}

Brandon D Spradley ${ }^{1}$, Kristy R Crowley ${ }^{1}$, Chih-Yin Tai ${ }^{1}$, Kristina L Kendall ${ }^{2}$, David H Fukuda ${ }^{2}$, Enrico N Esposito ${ }^{1}$, Sarah $\mathrm{EMoon}^{3}$ and Jordan R Moon ${ }^{1 *}$

\begin{abstract}
Background: The purpose of this study was to determine the effects of the pre-workout supplement AssaultTM (MusclePharm, Denver, CO, USA) on upper and lower body muscular endurance, aerobic and anaerobic capacity, and choice reaction time in recreationally-trained males. Subjective feelings of energy, fatigue, alertness, and focus were measured to examine associations between psychological factors and human performance.

Methods: Twelve recreationally-trained males participated in a 3-week investigation (mean +/- SD, age: $28+/-5 \mathrm{y}$, height: $178+/-9 \mathrm{~cm}$, weight: $\left.79.2+/-15.7 \mathrm{~kg}, V O_{2 \max }: 45.7+/-7.6 \mathrm{ml} / \mathrm{kg} / \mathrm{min}\right)$. Subjects reported to the human performance laboratory on three separate occasions. All participants completed a baseline/familiarization day of testing that included a maximal graded exercise test for the determination of aerobic capacity $\left(\mathrm{VO}_{2 \text { max }}\right)$, one-rep maximum (1-RM) for bench and leg press to determine $75 \%$ of 1-RM, choice reaction tests, and intermittent critical velocity familiarization. Choice reaction tests included the following: single-step audio and visual, one-tower stationary protocol, two-tower lateral protocol, three-tower multi-directional protocol, and three-tower multidirectional protocol with martial arts sticks. Subjects were randomly assigned to ingest either the supplement (SUP) or the placebo $(\mathrm{PL})$ during Visit 2. Subjects were provided with the cross-over treatment on the last testing visit. Testing occurred 20 min following ingestion of both treatments.

Results: Significant $(p<0.05)$ main effects for the SUP were observed for leg press (SUP: $13 \pm 6$ reps, PL: $11 \pm 3$ reps), perceived energy (SUP: $3.4 \pm 0.9$, PL: $3.1 \pm 0.8$ ), alertness (SUP: $4.0 \pm 0.7$, PL: $3.5 \pm 0.8$ ), focus (SUP: $4.1 \pm$ 0.6, PL: $3.5 \pm 0.8$ ), choice reaction audio single-step (SUP: $0.92 \pm 0.10$ s, PL: $0.97 \pm 0.11$ s), choice reaction multidirection $15 \mathrm{~s}$ (SUP: $1.07 \pm 0.12 \mathrm{~s}, \mathrm{PL}: 1.13 \pm 0.14 \mathrm{~s}$ ), and multi-direction for $30 \mathrm{~s}$ (SUP: $1.10 \pm 0.11 \mathrm{~s}, \mathrm{PL}: 1.14 \pm$ $0.13 \mathrm{~s}$ ).

Conclusions: Ingesting the SUP before exercise significantly improved agility choice reaction performance and lower body muscular endurance, while increasing perceived energy and reducing subjective fatigue. These findings suggest that the SUP may delay fatigue during strenuous exercise.
\end{abstract}

Keywords: Branch-chained amino acids, Recreationally-trained, Human performance

\footnotetext{
* Correspondence: jmoon@ussa.edu

'Department of Sports Fitness and Health, Human performance and body composition laboratory, United States Sports Academy, 1 Academy Drive, Daphne, AL 36526, USA

Full list of author information is available at the end of the article
} 


\section{Background}

Pre-workout supplementation has become a fundamental component in nutrition programs and a growing interest in the sports nutrition industry. Recent research indicates energy drinks are the most popular supplement next to multi-vitamins in the young adult population (18-35 year) [1]. Many athletes believe supplementation prior to training will result in greater focus, quicker reaction time, and increased power [1]. Performance-enhancing claims of dietary supplements have not been fully addressed in the context of sportspecific exercises. Therefore, examining the effects of nutritional supplements during an exercise training session has the potential to elucidate more practical recommendations and applications [2].

Most supplements contain different ingredients to produce ergogenic effects. When ingested together, these ingredients may work synergistically to enhance various aspects of exercise performance. The ergogenic effects of caffeine have been attributed to a number of possible mechanisms, primarily the blocking of adenosine receptors [3,4]. Caffeine inhibits action at adenosine receptors, which has been reported to decrease the perception of pain and effort, resulting in improved exercise performance $[5,6]$. Research has also suggested that adenosine-receptor antagonism contributes to improved performance via increases in neurotransmitter release and motor firing rate [3,7]. However, the effects of caffeine in combination with beta-alanine, branched chain amino acids (BCAAs), creatine, vitamin B-6, and vitamin B-12 on ratings of perceived effort and pain have not been well investigated, particularly when ingested as a pre-workout supplement. Both beta-alanine and creatine have been shown to delay the onset of neuromuscular fatigue and therefore potentially augment the ergogenic effect of caffeine [8,9]. Research has shown vitamin B-6 to play an important role in the metabolic pathways required for exercise [10], while vitamin B-12 assists with DNA synthesis, which is necessary for the formation of red blood cells [10]. Research suggests that when supplementing with BCAAs prior to physical activity, recovery improves due to an increase in protein synthesis and a reduction in protein degradation [11]. However, studies have indicated that BCAAs do not improve endurance performance [12]; therefore, further research is required to examine the effects of BCAA supplementation on various team sport-related performances (e.g. repeated sprint ability) [3].

Few studies have examined the effect of pre-workout supplements using protocols related to sports-specific training and team sport performance. Therefore, the purpose of this study was to determine the effects of a supplement containing BCAAs, creatine, beta-alanine, citruline malate, arginine, vitamin B-6, vitamin B-12, and caffeine (Assault ${ }^{\mathrm{TM}}$, MusclePharm, Inc., Denver, CO, USA) on upper and lower body muscular endurance, aerobic and anaerobic capacity, and choice reaction time.

\section{Methods \\ Participants}

Twelve recreationally-trained males participated in the three-week investigation (mean $+/-\mathrm{SD}$, age: $28+/-5 \mathrm{y}$, height: $178+/-9 \mathrm{~cm}$, weight: $79.15+/-15.7 \mathrm{~kg}, \mathrm{VO}_{2 \max }$ : $45.7+/-7.6 \mathrm{ml} / \mathrm{kg} / \mathrm{min})$. The investigation was approved by an Institutional Review Board for use of human subjects, and all subjects signed an informed consent and completed a health history questionnaire prior to the investigation. All subjects met the necessary inclusion requirements as assessed by a health history and exercise questionnaire. Subjects were required to have been free of any nutritional supplements or ergogenic aids for at least 6 weeks preceding the study and were asked to refrain from using any additional supplements during the course of the study. In addition, subjects regularly engaged in exercise activity on a consistent basis (workout days per week: $3.9+/-1.3$ days, workout hours per day: $1.7+/-1 \mathrm{~h}$, cardio training per week: $3.8+/-1.8 \mathrm{~h}$, resistance training per week: $4.3+/-1.8 \mathrm{~h}$ ). Subjects also reported participation in one or more of the following sport activities: running, weightlifting, swimming, cycling, tennis, baseball, basketball, football, kickboxing, lacrosse, and mixed martial arts.

\section{Study design}

This study used a randomized, double-blind, placebocontrolled cross-over design. Subjects reported to the human performance laboratory on three separate occasions. All participants completed a baseline/familiarization day of testing, including a maximal graded exercise test for the determination of aerobic capacity $\left(\mathrm{VO}_{2 \max }\right)$, one-rep maximum (1RM) for bench and leg press to determine $75 \%$ of $1 \mathrm{RM}$, choice reaction familiarization, which included single-step visual and audio protocol, one tower protocol, two tower lateral protocol, three tower multi-directional, three tower multi-direction protocol with martial arts stick, and intermittent critical velocity familiarization completed at $110 \%, 100 \%$, and $90 \%$ of $\mathrm{VO}_{2 \max }$. Subjects were instructed to refrain from performing any strenuous activity for $24 \mathrm{~h}$ before the subsequent testing sessions (Visit 2, 3). Subjects were also required to maintain their current training program throughout the duration of the study. Additionally, subjects were required to complete a 2-day food log before the second testing day. Two days prior to Visit 3, subjects were asked to consume the exact same foods/ 
beverages eaten and written in their 2-day food log as recorded prior to Visit 2. Subjects reported to the lab in a fasted state (12 h with ad libitum water) to ensure that any additional foods/beverages would not influence performance or negate the potential effects of the supplement (SUP) or placebo (PL). Following familiarization, subjects were randomly assigned to ingest either the SUP or PL for Visit 2 and were provided the crossover treatment on the last testing visit. Twenty minutes after ingestion, blood pressure and heart rate (HR) were re-assessed, and subjects completed a four-question survey (5-point likert scale) that asked them to describe their feelings of energy, fatigue, alertness, and focus at that particular moment [1]. Subjects were asked to rate their energy level, fatigue level, feelings of alertness, and feelings of focus using the following verbal anchors: $1=$ very low; 2 = low; 3 = average; $4=$ high; $5=$ very high. The questionnaire was administered immediately before each choice reaction testing session (Figure 1). Subjects ingested each supplement 20 min prior to testing since that is what is typically practiced by most recreational athletes.

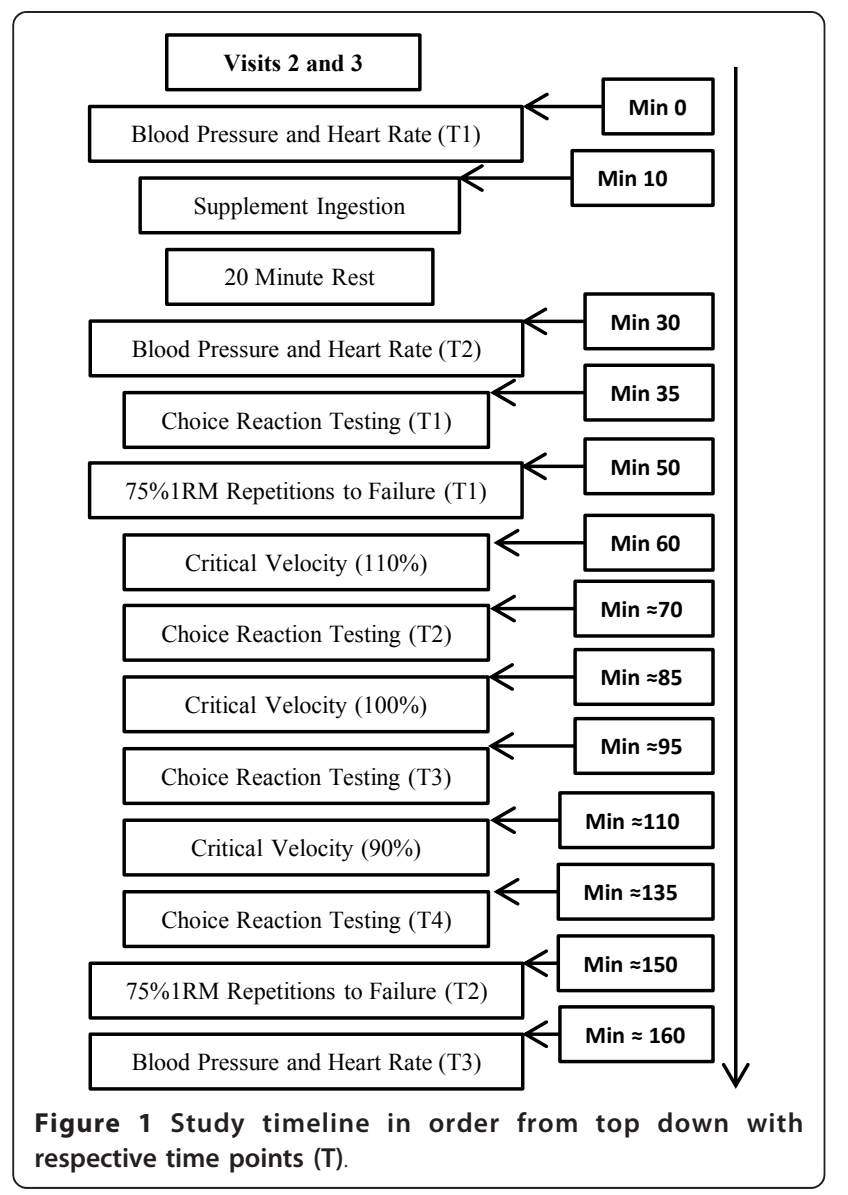

\section{Choice reaction test}

Reaction time was measured using the Makoto II Arena testing device (Makoto USA, Centennial, CO, USA). The Makoto II Arena device is constructed in the shape of a triangle that is $2.44 \mathrm{~m}$ from base to base and consists of three steel towers that are $1.83 \mathrm{~m}$ high with each tower containing 12 targets (Figure 2). Most studies that have investigated choice reaction time failed to utilize a testing device and protocol that is related to sport-specific training and team-sport performance. Due to the fact that most team sports require quick agile movements in different directions, the current investigation sought to utilize a testing device and protocol that measured stationary, lateral, and multi-directional movements. Each subject underwent nine different tests, measuring choice reaction time for each test. First, subjects were tested on a two-tower single step protocol. The reaction test consisted of audio (CRA) and visual (CRV) protocols, each completed separately. The decision to separate reaction time cues was made to accommodate the physiological transduction rate disparities between auditory and visual stimuli [13]. Targets used for this protocol included the middle target on each tower, located approximately at chest-level. The audio protocol consisted of only an auditory stimulus; therefore, a magnetic cover with the label $(\mathrm{X})$ was used to cover the specified target on each tower. The visual protocol consisted of only visual alerts, in which illumination of selected bulbs provided the stimulus. The volume was muted during this test, allowing subjects to use only their visual sense to detect which target illuminated. For

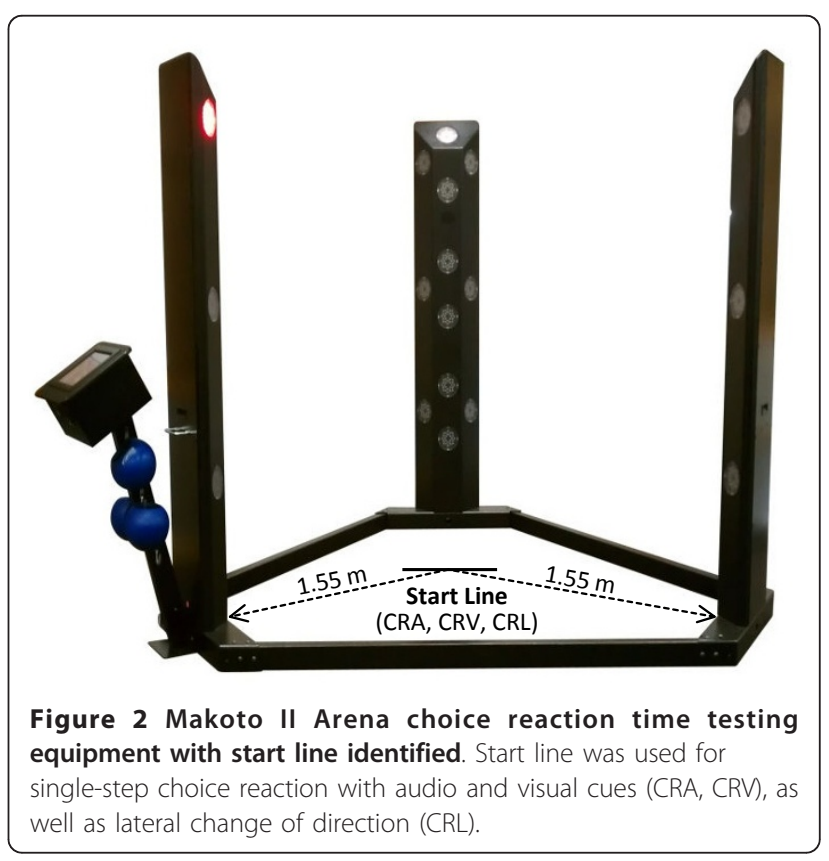


each test, subjects stood in the middle of the triangle behind a marked line located $1.55 \mathrm{~m}$ from both towers. Subjects stood behind the line with the third target (not being used) directly behind them (Figure 2). Subjects were required to lunge and make contact between their hand and the specified target on either tower. Built-in computer software randomly displayed the target on one of the two towers in the equivalent location.

Reaction time for each subject was calculated using built-in software and was recorded for each trial. Randomization was used to determine whether the subject would complete either the audio or visual protocol first. Subjects repeated each test for a total of three attempts, and the mean of the last two trials was used in analysis. Subjects then completed testing protocols at durations of $15 \mathrm{~s}$, using both audio and visual alerts. Testing procedures included one tower, for which the subjects remained stationary and only used their hands (CRS15), followed by two towers starting from the same line as CRV and CRA, for which subjects moved laterally from side to side (CRL15), and then three towers, for which subjects moved in multiple directions (CRM15); with two attempts for each test. The same testing procedures were followed again; only this time the duration was 30 s (CRS30, CRL30, CRM30). Mean reaction time for each trial was calculated internal to the device and recorded. The average of two back-to-back attempts was calculated and used for analysis. Skill levels indicated how long each target remained illuminated: the lower the level, the longer the target was lit. Each of the aforementioned tests was completed at the first skill level (level 1, $3 \mathrm{~s}$ count) to ensure each subject's ability to register a 'hit' with ease. Test-retest reliability of the above protocol calculated from six men at least 2 days apart yielded an ICC > 0.94 and a CV less than $10 \%$ for all choice reaction tests. Subjects completed the choice reaction testing protocol with a (three-tower) protocol using a large martial arts stick for two minutes. This protocol was set at the fourth skill level (1.20s count) to mimic the protocol used in a similar study by Hoffman et al. [1]. In our laboratory, test-retest reliability of the above protocol from six men at least 2 days apart yielded an $\mathrm{ICC}>0.81$. Average reaction time tested at different durations and different types of movements (stationary, lateral, multi-directional) allowed for a comprehensive analysis of agility compared to standard agility tests, such as the pro-shuttle and t-test for which agility speed, and not reaction time, is measured.

\section{Determination of $\mathrm{VO}_{2 \max }$}

All $\mathrm{VO}_{2 \max }$ tests were performed on a calibrated treadmill (Woodway Desmo, Waukesha, WI) and measured by indirect calorimetry using a metabolic cart (Parvomedics True One 2300, Sandy, UT, USA). Testing began with a three-minute warm-up stage at $5.15 \mathrm{~km} / \mathrm{h}$, while the grade remained at $0 \%$ throughout testing. Velocity increased to $10 \mathrm{~km} / \mathrm{h}$ for the next stage, then increased $2 \mathrm{~km} / \mathrm{h}$ for every two minutes up to $16 \mathrm{~km} / \mathrm{h}$, followed by $1 \mathrm{~km} / \mathrm{h}$ increments per minute up to $24 \mathrm{~km} / \mathrm{h}$. $\mathrm{VO}_{2 \max }$ was confirmed if at least two of the following criteria were met: (a) plateau in $\mathrm{HR}$ or $\mathrm{HR}$ values within $10 \%$ of the age-predicted HRmax, (b) plateau in $\mathrm{VO}_{2}$ (defined by an increase of no more than $150 \mathrm{ml} \cdot \mathrm{min}^{-1}$ ), (c) respiratory exchange ratio (RER) value greater than 1.15 .

\section{Intermittent critical velocity and intermittent anaerobic running capacity}

Intermittent Critical Velocity (iCV) testing protocols were similar to previous studies $[2,14]$. All iCV tests were performed on a calibrated treadmill (Woodway Desmo, Waukesha, WI, USA). Subjects completed three separate treadmill running tests at intervals of $10 \mathrm{~s}$ of running and $10 \mathrm{~s}$ of rest at $110 \%, 100 \%$, and $90 \%$ of their peak velocity at $\mathrm{VO}_{2 \max }$. A 25 -minute break period was given between running tests. During this break period, subjects performed the choice reaction protocol followed by approximately five minutes of inactivity before the next treadmill test. Each iCV testing session was terminated when the subject could no longer complete a 10 s sprint. Distance and total running time were recorded for each participant, and $\mathrm{iCV}$ and intermittent anaerobic running capacity (iARC) were calculated using the following equation: $($ Total Distance $=\mathrm{iARC}+\mathrm{iCV}$. total running time). An investigation by Fukuda et al., 2009 [14] examined the reliability of iCV testing in men and women, producing an ICC value of 0.87 and 0.83 .

\section{Muscular strength and endurance}

One-repetition maximum bench press and leg press were used to determine the maximum amount of weight a subject could lift one time for each exercise. Subjects performed one warm-up set for bench and leg press $(\approx 50 \%$ of estimated 1RM) and rested for two minutes between attempts. Resistance was increased until the participant failed to complete a repetition; subjects performed no more than five attempts. Maximum strength for bench and leg press exercises was measured using plate-loaded iso-lateral bench and leg press (Hammer Strength, Cincinnati, $\mathrm{OH}, \mathrm{USA}$ ). Seat height for bench and leg press was adjusted for each subject and remained consistent throughout the course of the study. A repetition was recorded when subjects lowered the weight in an eccentric contraction and then pushed the weight in a concentric contraction with full joint extension, ensuring the weights made contact with the back bumper pads after eccentric movement. Following 1RM testing, 75\% of each subject's $1 \mathrm{RM}$ was calculated, and repetitions to fatigue were 
assessed and recorded at respective weight resistance on subsequent testing visits. Subjects were required to perform a set (at least 5 repetitions) of $75 \%$ of their 1RM for familiarization on Visit 1.

\section{Supplement}

On the second and third testing days, subjects consumed 1 scoop (2 servings, $46 \mathrm{~g}$ ) of either the PL or SUP powder mixed with $\approx 16 \mathrm{oz}$. of water. The SUP, marketed as Assault ${ }^{\mathrm{TM}}$ (MusclePharm, Denver, Colorado, USA), contained BCAAs ( $6 \mathrm{~g})$, creatine $(5 \mathrm{~g})$, betaalanine ( $4 \mathrm{~g})$, citruline malate $(1.5 \mathrm{~g})$, and caffeine $(300$ $\mathrm{mg})$. Complete nutritional facts are listed in Figure 3. The PL was flavored maltodextrin, similar in color, taste and flavor to the SUP formulation. An investigator secluded from any data collection prepared and administered both supplement and placebo beverages for all subjects, using the same amount of water and powder for each beverage. Subjects were provided cold water ad libitum throughout the study.

\section{Data analysis}

All recurring tests were analyzed using a repeated measure analysis of variance (ANOVA) [time $(\mathrm{T} 1-\mathrm{T} 4) \times$

\begin{tabular}{|c|}
\hline $\begin{array}{l}\text { Supplement Facts } \\
\text { Serving Size } 23 \mathrm{~g}(1 / 2 \text { scoop }) \\
\text { Servings Per Container: } 32\end{array}$ \\
\hline Amount Per Serving \\
\hline Calories \\
\hline Total Carbohydrates \\
\hline Sugars \\
\hline Vitamin B-6 (Pyridoxine HCL) \\
\hline Vitamin B-12 (Methylcobalamin) \\
\hline Calcium (from Phosphate and Silicate) \\
\hline Sodium \\
\hline Potassium \\
\hline Assault Proprietary Blend \\
\hline $\begin{array}{l}\text { Anti-Fatigue Adaption Matrix } \\
\text { CarnoSyn }{ }^{\star} \text { Beta-Alanine, Citrulline Malate, (DMG) Dimethylglycine, Rhodiola } \\
\text { Rosea (Root) Extract } 3 \% \text { Rosavins }\end{array}$ \\
\hline $\begin{array}{l}\text { Strength Domination \& Recovery Matrix } \\
\text { Creatine Monohydrate, Con-Cret } \Theta \text {, Creatine } \mathrm{HCl}(100 \% \text { Concentrated } \\
\text { Creatine) }\end{array}$ \\
\hline $\begin{array}{l}\text { High Performance Energy \& Intensity Amplifier } \\
\text { Choline Bitartrate, Taurine, L-Tyrosine, Medium Chain Triglycerides (MCT), } \\
\text { Caffeine Anhydrous, Papain 1000, Pyroglutamic Acid, L-Aspartic Acid, } \\
\text { Camellia sinensis (White Tea Leaf Powder), Pyridoxine HCl, Methylcobalamin }\end{array}$ \\
\hline $\begin{array}{l}\text { 3:1:2 BCAA Ratio (Patent Pending) } \\
\text { BCAAs (L-Leucine, L-Valine, L-Isoleucine) }\end{array}$ \\
\hline $\begin{array}{l}\text { Cell Volumizer } \\
\text { L-Glycine, L-Arginine Blend (L-Arginine AKG 2:1, Di Arginine Malate, } \\
\text { L-Arginine HCL) }\end{array}$ \\
\hline $\begin{array}{l}\text { Cellular Transport Amplifier } \\
\text { Astragin } \AA \text { (Notoginseng Root Ext, Astragalus Root Ext.), Cinnulin PF® } \\
\text { (Cinnamomum burmanii extract standardized to Type-A polymers) }\end{array}$ \\
\hline $\begin{array}{l}\text { Muscle Hydration \& Electrolyte Blend } \\
\text { L-Glutamine, Phosphorus, Sodium, Potassium }\end{array}$ \\
\hline $\begin{array}{l}\text { Other Ingredients } \\
\text { Glucose Polymers, Natural and Artificial Flavors, Calcium Silicate, Citric Acid, } \\
\text { Sucralose, Acesulfame Potassium, Salt, Calcium Phosphate, Magnesium } \\
\text { Oxide, Potassium Chloride, FD\&C Red\#40 }\end{array}$ \\
\hline $\begin{array}{l}\text { TPercent Daily Values are based on a 2,000 calorie diet. } \\
\text { "Daily Value not established. }\end{array}$ \\
\hline
\end{tabular}

drink (SUP vs. PL)]. Significant interactions between time and supplement were analyzed using post-hoc dependent t-tests. In addition, iCV and iARC were analyzed using dependent t-tests. Significant main effects for time and for supplement were analyzed using Bonferroni post-hoc tests to account for multiple comparisons by maintaining family-wise error rates. A p-value less than 0.05 was considered significant for this investigation. All ANOVA assumptions were met, and analyses were performed using the computer program SPSS (PASW Statistics 18.0.0, IBM Corporation, Armonk, NY, USA).

\section{Results}

Interactions were observed at time point 3 (T3) for heart rate (HR) (SUP: $102+/-12$, PL: $94+/-15 ; p<$ 0.05), energy (SUP: $3.6+/-0.8$, PL: $2.7+/-0.5 ; p<$ 0.05), fatigue (SUP: $2.6+/-0.8$, PL: $3.7+/-0.7 ; p<0.05$ ) and CRM15 (SUP: $1.03+/-0.11$, PL: $1.19+/-0.04 ; p<$ 0.05. Main effects for time were observed for systolic blood pressure, HR, leg press repetitions, energy, fatigue, alertness, focus, choice reaction visual single step (CRV), choice reaction multi-direction $30 \mathrm{~s}$ (CRM30), and stick hits for the two-minute protocol $(p<0.05)$. Post-hoc results for time were independent of the supplement and, therefore, are not reported. Significant main effects for the supplement treatment were observed for leg press repetitions (SUP: $13+/-6$ reps, PL: $11+/-3$ reps; $p<0.05$ ), energy (SUP: $3.4+/-0.9$, PL: $3.1+/-0.8 ; p<$ 0.05 ), alertness (SUP: $4.0+/-0.7$, PL: $3.6+/-0.8$; $p<$ 0.05 ), and focus (SUP: $4.1+/-0.6$, PL: $3.6+/-0.8$; $p<$ 0.05). No significant differences were seen comparing the SUP to PL for iCV (PL: $3.11+/-1.2$, SUP: $3.15+/$ $1.2 \mathrm{~m} / \mathrm{s}, p=0.95)$ or iARC (PL: $318.8+/-408.7$, SUP: $263.3+/-416.3 \mathrm{~m}, p=0.78$ ). Therefore, no significant differences were apparent for total treadmill work between testing days. Results are depicted in Figures 4, 5 and 6 and choice reaction results are presented in Table 1.

\section{Discussion}

This investigation was the first to examine the effects of the pre-workout supplement Assault ${ }^{\mathrm{TM}}$ on a multifaceted, exercise testing protocol. The results suggest that the pre-workout supplement significantly improved muscular endurance and choice reaction time.

The primary active ingredient in Assault ${ }^{\mathrm{TM}}$ is caffeine. Caffeine is a mild stimulant that affects the central nervous system and has the potential to influence human neuromuscular performance. In an attempt to maximize the effectiveness of caffeine, supplement manufacturers often combine several ingredients, possibly enhancing caffeine's stimulatory potential [1]. A study conducted by Fukuda et al. [15], showed an acute anaerobic effect 


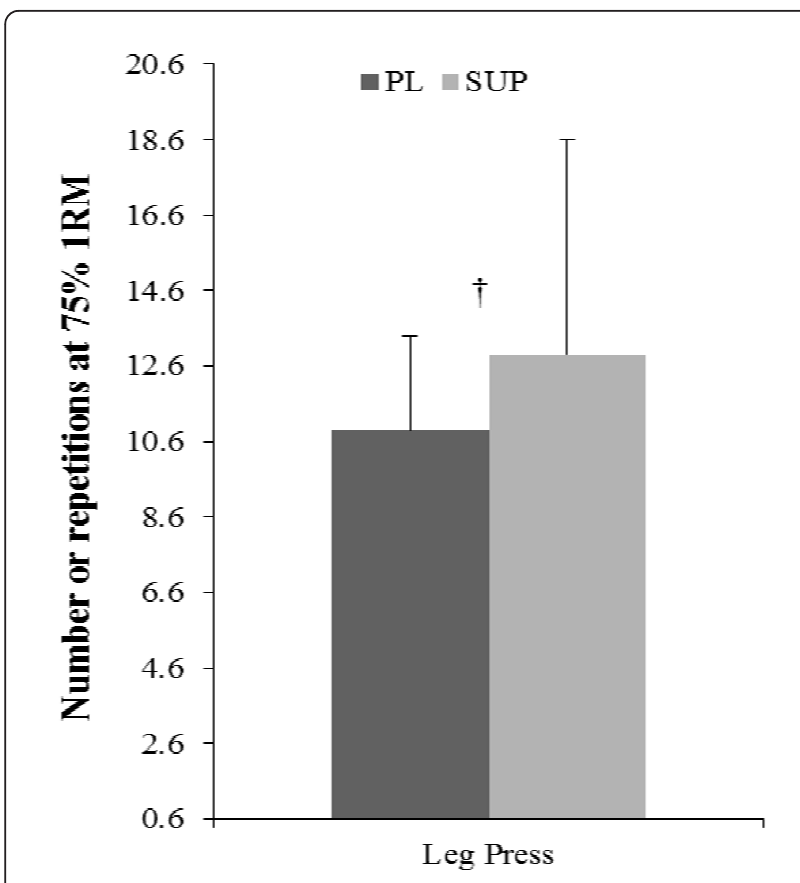

Figure 4 Total leg press repetitions at $75 \%$ of one repetition maximum. $+=$ Significant difference between SUP and $P L, p<$ 0.05. PL = Placebo, SUP = Supplement (AssaultTM).

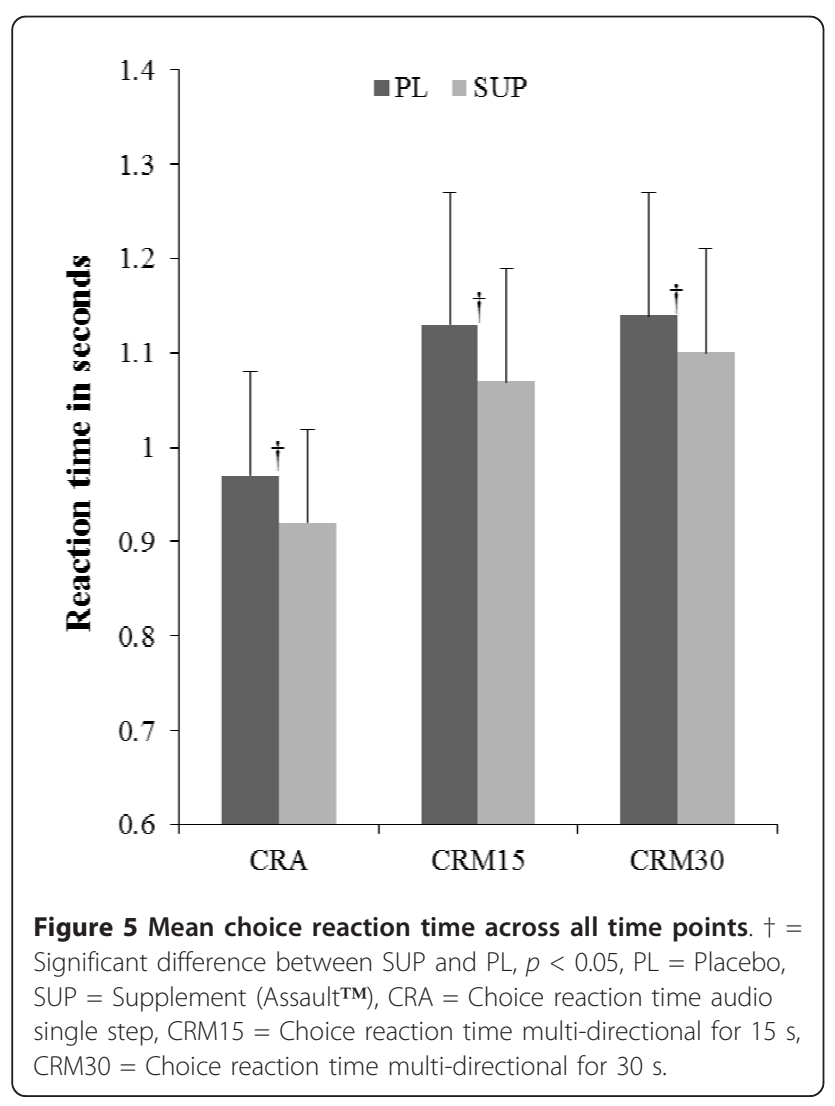

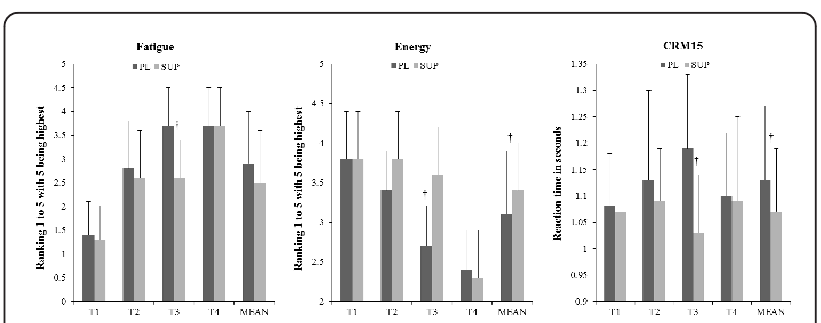

Figure 6 Fatigue, energy, and choice reaction time comparing means and time points. $\dagger=$ Significant difference between SUP and $\mathrm{PL}, p<0.05, \mathrm{PL}=$ Placebo, SUP $=$ Supplement (Assault $\mathrm{TM}), \mathrm{T}=$ time point, CRM15 = Choice reaction time multi-directional for $15 \mathrm{~s}$.

in men and women with the ingestion of a low dosage of caffeine, BCAAs, and creatine, which could possibly lead to improvements in various aspects of human performance (muscle strength, power, etc).

In the current investigation, a $26.5 \%$ reduction in total repetitions ( 3 reps) at 75\% of 1RM was observed from time point 1 to time point 2 in the PL group for leg press, while an $11.7 \%$ ( 2 reps) reduction from time point 1 to time point 2 was observed in the SUP treatment for leg press. Results also indicated positive effects on perceived alertness, energy, and focus, which may have contributed to a $4 \%$ improvement in CRA and CRM30, and a 5\% improvement in CRM15. These findings are consistent with previous studies $[16,17]$ that also reported significant improvements in choice reaction time following the ingestion of a supplement containing $5-6 \mathrm{mg} / \mathrm{kg}$ of caffeine, which is higher than the amount used in the current study (mean $=3.8+/-0.7 \mathrm{mg} / \mathrm{kg}$ ).

There are many possible reasons for the improvements in performance demonstrated in this study. At the cellular level, caffeine enhances neuromuscular transmission and improves skeletal muscle contractility [18]. Green et al. [19] investigated the effects of caffeine $(6 \mathrm{mg} / \mathrm{kg})$ on lower body muscular endurance with repetitions performed to volitional failure. Results indicated that caffeine was associated with significantly higher repetitions $(12.5 \pm 4.2,9.9 \pm 2.6)$ during the third set of leg press exercises compared to PL [19]. In agreement, the current investigation showed significant increases in both total leg press repetitions (SUP $13 \pm 6$, PL $11 \pm 3$, $p=0.021$ ) at T1 and T2 (SUP $12 \pm 5$, PL $8 \pm 6, p=$ $0.024)$, although no significant time and SUP interaction was found (Figure 4).

A study by Barry et al. [20] examining caffeineinduced arousal effects on performance and auditory event-related potentials in the brain, indicated a reduction in reaction time and suggested that caffeine differentially improves processing aspects related to task performance [20]. The decrease in CRA times may have had an effect on the improvement in CRM15 and CRM30 because they also include an auditory 
Table 1 Choice Reaction Time Results

\begin{tabular}{|c|c|c|c|c|c|c|}
\hline Variable & Treatment & $\mathrm{T} 1$ & $\mathrm{~T} 2$ & T3 & T4 & Mean \\
\hline \multirow[t]{2}{*}{ Single Step Visual (CRV) } & $\mathrm{PL}$ & $0.97 \pm 0.14$ & $0.98 \pm 0.25$ & $0.94 \pm 0.11$ & $0.92 \pm 0.15$ & $0.95 \pm 0.17$ \\
\hline & SUP & $0.98 \pm 0.23$ & $0.93 \pm 0.14$ & $0.91 \pm 0.16$ & $0.88 \pm 0.12$ & $0.93 \pm 0.16$ \\
\hline \multirow{2}{*}{$\begin{array}{l}\text { Single Step Audio } \\
\text { (CRA) }\end{array}$} & $\mathrm{PL}$ & $0.98 \pm 0.09$ & $0.96 \pm 0.13$ & $0.96 \pm 0.11$ & $0.95 \pm 0.12$ & $0.97 \pm 0.11$ \\
\hline & SUP & $0.96 \pm 0.10$ & $0.91 \pm 0.09$ & $0.89 \pm 0.07$ & $0.92 \pm 0.11$ & $0.92 \pm 0.10^{\dagger}$ \\
\hline \multirow{2}{*}{$\begin{array}{l}\text { Stationary } 15 \mathrm{~s} \\
\text { (CRS15) }\end{array}$} & $P L$ & $0.54 \pm 0.04$ & $0.54 \pm 0.07$ & $0.56 \pm 0.09$ & $0.55 \pm 0.08$ & $0.55 \pm 0.07$ \\
\hline & SUP & $0.56 \pm 0.06$ & $0.51 \pm 0.05$ & $0.51 \pm 0.06$ & $0.51 \pm 0.06$ & $0.52 \pm 0.06$ \\
\hline \multirow[t]{2}{*}{ Lateral 15 s (CRL15) } & $\mathrm{PL}$ & $0.91 \pm 0.11$ & $0.91 \pm 0.15$ & $0.95 \pm 0.11$ & $0.89 \pm 0.13$ & $0.91 \pm 0.13$ \\
\hline & SUP & $0.93 \pm 0.11$ & $0.86 \pm 0.07$ & $0.88 \pm 0.11$ & $0.89 \pm 0.12$ & $0.89 \pm 0.10$ \\
\hline \multirow[t]{2}{*}{ Multi-directional $15 \mathrm{~s}$ (CRM15) } & $\mathrm{PL}$ & $1.08 \pm 0.10$ & $1.13 \pm 0.17$ & $1.19 \pm 0.14$ & $1.10 \pm 0.12$ & $1.13 \pm 0.14$ \\
\hline & SUP & $1.07 \pm 0.09$ & $1.09 \pm 0.10$ & $1.03 \pm 0.11^{\ddagger}$ & $1.09 \pm 0.16$ & $1.07 \pm 0.12^{t}$ \\
\hline \multirow[t]{2}{*}{ Stationary 30s (CRS30) } & $\mathrm{PL}$ & $0.56 \pm 0.07$ & $0.55 \pm 0.09$ & $0.54 \pm 0.09$ & $0.54 \pm 0.07$ & $0.55 \pm 0.08$ \\
\hline & SUP & $0.55 \pm 0.05$ & $0.52 \pm 0.07$ & $0.52 \pm 0.06$ & $0.52 \pm 0.07$ & $0.53 \pm 0.06$ \\
\hline \multirow[t]{2}{*}{ Lateral 30s (CRL30) } & $\mathrm{PL}$ & $0.89 \pm 0.10$ & $0.93 \pm 0.13$ & $0.92 \pm 0.10$ & $0.93 \pm 0.15$ & $0.92 \pm 0.12$ \\
\hline & SUP & $0.91 \pm 0.10$ & $0.91 \pm 0.11$ & $0.87 \pm 0.12$ & $0.90 \pm 0.10$ & $0.90 \pm 0.11$ \\
\hline \multirow[t]{2}{*}{ Multi-directional 30s (CRM30) } & $\mathrm{PL}$ & $1.15 \pm 0.13$ & $1.16 \pm 0.12$ & $1.11 \pm 0.15$ & $1.15 \pm 0.12$ & $1.14 \pm 0.13$ \\
\hline & $\overline{\text { SUP }}$ & $1.11 \pm 0.10$ & $1.10 \pm 0.12$ & $1.06 \pm 0.12$ & $1.12 \pm 0.12$ & $1.10 \pm 0.11^{1}$ \\
\hline \multirow[t]{2}{*}{ Stick Hits (2 min) } & $\mathrm{PL}$ & $63 \pm 11$ & $67 \pm 10$ & $63 \pm 11$ & $61 \pm 12$ & $63 \pm 11$ \\
\hline & SUP & $66 \pm 7$ & $70 \pm 10$ & $65 \pm 11$ & $65 \pm 12$ & $66 \pm 10$ \\
\hline
\end{tabular}

PL placebo; SUP supplement; $T$ time point; $\dagger=$ significant main effect for supplement, $p<0.05 ; \ddagger=$ significant interaction between time and supplement, $p<$ 0.05 ; All data are reported as mean \pm standard deviation.

component. Figure 5 illustrates that the reduction in multi-directional choice reaction time (CRM) for $15 \mathrm{~s}$ and $30 \mathrm{~s}$ was most likely due to the significant decrease in choice reaction time using an auditory stimulus (CRM15, SUP: $1.07 \pm 0.12 \mathrm{~s}$, PL: $1.13 \pm 0.14 \mathrm{~s}, p=$ 0.007; CRM30, SUP: $1.10 \pm 0.11 \mathrm{~s}$, PL: $1.14 \pm 0.13 \mathrm{~s}, p=$ 0.013). Caffeine's stimulatory effects on the central nervous system seem to manifest in performance in activities which require quick reactions and movements [18]. Thus, it is evident that choice reaction time improved following the ingestion of $3.8+/-0.7 \mathrm{mg} / \mathrm{kg}$ of caffeine (approx. $300 \mathrm{mg}$ ) for recreationally-trained males between the ages of 18 and 35 years. It remains unknown as to why the single-tower and two-tower reaction times were not significantly different between groups, as they included both audio and visual cues. It is hypothesized that the three-tower, multi-directional reaction time tests required the greatest movement and consequently the greatest task difficulty and overall mental and physical demand; thus, fatigue played a larger role in reaction time compared to the single- and two -tower tests. Independent of fatigue, caffeine could have influenced the multi-directional reaction tests because of the increased task complexity. Therefore, the improvements in the three-tower, multi-directional reaction time protocol after supplementation could have been attributed to both a reduction in fatigue as well as enhanced cognitive function. More research is required to determine the influence of the SUP used in the current investigation on reaction times using audio versus visual cues and various sport-specific movements.

An investigation by Fukuda et al. [15] examined whether a pre-workout supplement with similar ingredients (caffeine, creatine, amino acids) to the SUP used in the present study would impact anaerobic running capacity and critical velocity. Results showed no significant different between the SUP and the PL for critical velocity. However, it was reported that the SUP significantly increased anaerobic running capacity compared to the PL. In the present study, no significant differences were seen in anaerobic running capacity or intermittent critical velocity with the ingestion of the pre-workout supplement. However, the study by Fukuda et al. [15] was conducted in college-aged men and women and indicated an overall increase in anaerobic running capacity of $10.8 \%$ compared to the placebo with an increase in time to exhaustion at $110 \%, 105 \%$, and $100 \%$ of peak velocity. Suggesting, the multi-ingredient supplement used in the investigation had effects on anaerobic performance only, which is similar to the current findings. Differences between findings could be related to the subjects and protocols used in the investigations. More research is needed regarding continuous anaerobic running capacity, critical velocity, and pre-workout 
supplements containing similar ingredients to those found in the SUP.

The SUP treatment decreased fatigue, increased energy, and improved choice reaction time (CRM15). These data suggest that at time point three (T3), around 95 min into the exercise protocol, as the subjects began to fatigue, the SUP caused a delay in fatigue and an increase in energy as evidenced by significantly faster choice reaction times (CRM15). Therefore, it appears that a single scoop ( 2 doses) of the SUP taken $20 \mathrm{~min}$ prior to exercise or training could allow for more total work with a higher quality (faster reactions) of work. Additionally, across all choice reaction time tests, mean values were faster for the SUP compared to the PL during time points two, three, and four with the exception of CRL15 at time point four, where the means were the same. At time point one; $20 \mathrm{~min}$ after ingestion, before any other exercises were completed, the SUP produced faster reactions in only four of the eight reaction tests. These findings suggest that the SUP began to effect performance only after the first round of testing, suggesting the largest factor related to improved performance was the ability of the SUP to delay fatigue and maintain high levels of energy.

Some limitations of this investigation were the use of lower percentages of speed at $\mathrm{VO}_{2 \max }$ to measure iCV and iARC compared to other investigations [14], which could have reduced the ability to find significant changes in anaerobic running performance, although it is more likely that a single dose of the SUP had little or no effect on iCV or iARC because creatine and beta alanine both require loading or prolonged use to see performance benefits, particularly in iCV or iARC.

Also, the fact that the SUP had multiple ingredients makes it impossible to identify which specific ingredients contributed to improved performance. Beta-alanine and creatine, two of the active ingredients in the preworkout supplement (MusclePharm Assault ${ }^{\mathrm{TM}}$ ) have both been shown to improve anaerobic capacity $[8,21]$. Most studies that investigated the effects of beta-alanine on exercise performance reported an increase in muscle carnosine [9,21,22]; although, those studies incorporated beta-alanine loading, stating that a longer supplementation period ( $\geq 4$ weeks) could produce greater increases in muscle carnosine. In theory, a significant increase in muscle carnosine could result in an increase in muscle buffering capacity [22], translating to improvements in anaerobic running capacities by decreasing the accumulation of hydrogen ions. In the present study, no significant differences were seen in anaerobic running capacity and intermittent critical velocity with the one-time ingestion of the pre-workout supplement. Creatine and beta-alanine both require loading periods of several weeks to positively influence exercise performance. As a result, the only active ingredients likely to influence performance in this investigation were caffeine and Bvitamins.

Study limitations also relate to the characteristics of the subjects who participated in the current investigation. While the men were recreationally-trained with strength values in the $75^{\text {th }}$ and $90^{\text {th }}$ percentile (ACSM guidelines) for bench and leg press $1 \mathrm{RM}$ and $\mathrm{VO}_{2 \max }$ values in the $60^{\text {th }}$ percentile, the SUP may not have the same effects in men who are more anaerobically- or aerobically-trained. Nonetheless, the subjects in the current study should represent a large population of active men who regularly exercise. In addition, two-day food logs indicated subjects consumed an average of $31.5+/$ $109.4 \mathrm{mg}$ of caffeine per day in their normal diet, and active men who regularly consume larger quantities of caffeine may not experience the same results as the men in the current investigation.

\section{Conclusion}

The overall findings of this investigation indicate that the supplement Assault ${ }^{\mathrm{TM}}$ can significantly improve perceived feelings of energy, focus, and alertness leading to a significant improvement in multi-directional choice reaction time and single-step choice reaction time with an auditory stimulus. Results also support that the SUP may reduce both feelings of fatigue and actual fatigue, which can result in performance increases in both muscular endurance and reaction time. Additionally, from a practical perspective, college-age males who recreationally participate in sports where auditory stimuli are abundant, such as team sports where verbal cues are encouraged may experience increases in performance after ingesting the pre-workout supplement used in the current study (Assault ${ }^{\mathrm{TM}}$ ).

\section{Acknowledgements}

We would like to thank all of the men who participated and MusclePharm, Inc. for supplying the products and funding the investigation.

\section{Author details}

${ }^{1}$ Department of Sports Fitness and Health, Human performance and body composition laboratory, United States Sports Academy, 1 Academy Drive, Daphne, AL 36526, USA. ²Department of Health and Exercise Science, Metabolic and body composition laboratory, University of Oklahoma, Norman, OK, USA. ${ }^{3}$ University of South Alabama, College of Medicine, Mobile, AL, USA.

\section{Authors' contributions}

$B S, K C, C T, K K, D F, E E$, and $J M$ participated in the study design and helped draft the manuscript while aiding in data analysis. BS, KC, CT, and EE participated in data collection. Additionally, all authors read and approved the final manuscript.

\section{Competing interests}

All authors have no financial interests concerning the outcome of this investigation. This publication should not be viewed as endorsement by the investigators, the United States Sports Academy, the University of Oklahoma, or the Journal of the Nutrition \& Metabolism. 
Received: 21 December 2011 Accepted: 30 March 2012

Published: 30 March 2012

\section{References}

1. Hoffman JR, Kang J, Ratamess NA, Hoffman MW, Tranchina CP, Faigenbaum AD: Examination of a pre-exercise, high energy supplement on exercise performance. Journal of the International Society of Sports Nutrition 2009, 6:2

2. Smith AE, Fukuda DH, Kendall KL, Stout JR: The effects of a pre-workout supplement containing caffeine, creatine, and amino acids during three weeks of high-intensity exercise on aerobic and anaerobic performance. Journal of the International Society of Sports Nutrition 2010, 7:10.

3. Bishop D: Dietary supplements and team-sport performance. Sports medicine 2010, 40:995-1017.

4. Fredholm BB, Battig K, Holmen J, Nehlig A, Zvartau EE: Actions of caffeine in the brain with special reference to factors that contribute to its widespread use. Pharmacological reviews 1999, 51:83-133.

5. Doherty M, Smith PM: Effects of caffeine ingestion on exercise testing: a meta-analysis. International journal of sport nutrition and exercise metabolism 2004, 14:626-646.

6. Sokmen B, Armstrong LE, Kraemer WJ, Casa DJ, Dias JC, Judelson DA, Maresh CM: Caffeine use in sports: considerations for the athlete. Journal of strength and conditioning research 2008, 22:978-986.

7. Graham TE: Caffeine and exercise: metabolism, endurance and performance. Sports medicine 2001, 31:785-807.

8. Snow RJ, McKenna MJ, Selig SE, Kemp J, Stathis CG, Zhao S: Effect of creatine supplementation on sprint exercise performance and muscle metabolism. Journal of applied physiology 1998, 84:1667-1673.

9. Derave W, Ozdemir MS, Harris RC, Pottier A, Reyngoudt H, Koppo K, Wise JA, Achten E: Beta-Alanine supplementation augments muscle carnosine content and attenuates fatigue during repeated isokinetic contraction bouts in trained sprinters. Journal of applied physiology 2007 103:1736-1743.

10. Woolf $\mathrm{K}$, Manore MM: B-vitamins and exercise: does exercise alter requirements? International journal of sport nutrition and exercise metabolism 2006, 16:453-484.

11. Blomstrand E, Eliasson J, Karlsson HK, Kohnke R: Branched-chain amino acids activate key enzymes in protein synthesis after physical exercise. The Journal of nutrition 2006, 136:269S-273S.

12. Meeusen R, Watson P, Dvorak J: The brain and fatigue: new opportunities for nutritional interventions? Journal of sports sciences 2006, 24:773-782.

13. Recanzone GH: Interactions of auditory and visual stimuli in space and time. Hearing research 2009, 258:89-99.

14. Fukuda DH, Smith AE, Kendall KL, Hetrick RP, Hames RL, Cramer JT, Stout JR The reliability of the intermittent critical velocity test and assessment of critical rest interval in men and women. Eur J Appl Physiol Occup Physiol 2011, 112:1197-1205

15. Fukuda DH, Smith AE, Kendall KL, Stout JR: The possible combinatory effects of acute consumption of caffeine, creatine, and amino acids on the improvement of anaerobic running performance in humans. Nutrition research 2010, 30:607-614.

16. Jacobson BH, Edgley BM: Effects of caffeine on simple reaction time and movement time. Aviation, space, and environmental medicine 1987, 58:1153-1156.

17. Kruk B, Chmura J, Krzeminski K, Ziemba AW, Nazar K, Pekkarinen H, KaciubaUscilko H: Influence of caffeine, cold and exercise on multiple choice reaction time. Psychopharmacology 2001, 157:197-201.

18. Williams JH: Caffeine, neuromuscular function and high-intensity exercise performance. The Journal of sports medicine and physical fitness 1991, 31:481-489.

19. Green JM, Wickwire PJ, McLester JR, Gendle S, Hudson G, Pritchett RC, Laurent CM: Effects of caffeine on repetitions to failure and ratings of perceived exertion during resistance training. International journal of sports physiology and performance 2007, 2:250-259.

20. Barry RJ, Johnstone SJ, Clarke AR, Rushby JA, Brown CR, Mckenzie DN: Caffeine effects on ERPs and performance in an auditory Go/NoGo task. Clinical neurophysiology 2007, 118:2692-2699.

21. Hill CA, Harris RC, Kim HJ, Harris BD, Sale C, Boobis LH, Kim CK, Wise JA: Influence of beta-alanine supplementation on skeletal muscle carnosine concentrations and high intensity cycling capacity. Amino acids 2007, 32:225-233.
22. Kendrick IP, Harris RC, Kim HJ, Kim CK, Dang VH, Lam TQ, Bui TT, Smith M, Wise JA: The effects of 10 weeks of resistance training combined with beta-alanine supplementation on whole body strength, force production, muscular endurance and body composition. Amino acids 2008, 34:547-554.

doi:10.1186/1743-7075-9-28

Cite this article as: Spradley et al:: Ingesting a pre-workout supplement containing caffeine, B-vitamins, amino acids, creatine, and beta-alanine before exercise delays fatigue while improving reaction time and muscular endurance. Nutrition \& Metabolism 2012 9:28.

\section{Submit your next manuscript to BioMed Central and take full advantage of:}

- Convenient online submission

- Thorough peer review

- No space constraints or color figure charges

- Immediate publication on acceptance

- Inclusion in PubMed, CAS, Scopus and Google Scholar

- Research which is freely available for redistribution

Submit your manuscript at www.biomedcentral.com/submit
Ciomed Central 